\title{
Supernova 1986J: a Neutron Star or Black Hole in the Centre?
}

\author{
Michael F. Bietenholz ${ }^{1,2}$ \\ ${ }^{1}$ Hartebeesthoek Radio Observatory, PO Box 443, Krugersdorp, 1740, South Africa. ${ }^{2}$ Also at \\ Department of Physics and Astronomy, York University, Toronto, M3J 1P3, Ontario, Canada.
}

\begin{abstract}
Supernova 1986J is almost the same age as SN 1987A, but was Type IIn, and likely had a massive progenitor. Located at $10 \mathrm{Mpc}$ in NGC 891, it is one of the few supernovae whose radio emission can be resolved using VLBI. We present a new 5-GHz global-VLBI image of SN 1986J from 2014 as well as broadband VLA flux-density measurements. SN 1986J is unusual in that a compact synchrotron radio-emitting component appeared in the centre of the expanding shell of ejecta $\sim 14$ yr after the explosion, which now dominates the VLBI image. The central component is stationary to within the uncertainties $\left(<570 \mathrm{~km} \mathrm{~s}^{-1}\right)$, and it has a marginally resolved HWHM radius of $\left(6.7_{-3.7}^{+0.7}\right) \times 10^{16} \mathrm{~cm}$. The shell has expanded with average $v \simeq 5400 \mathrm{~km} \mathrm{~s}^{-1}$. The central component's 5-GHz flux density is still increasing with time, and at present it has a 5-GHz $\nu L_{\nu}$ luminosity of $\sim 4 \times 10^{35} \mathrm{erg} \mathrm{s}^{-1}, \sim 20$ times that of the Crab Nebula. The central component may be due to a newly formed pulsar wind nebula, or an accreting black hole, or it may be due to interaction of the supernova shock with a highly structured environment left over from a progenitor which was in a close binary system. We discuss the newest observations and the constraints on its nature.
\end{abstract}

Keywords. (stars:) supernovae: individual (SN 1986J), radio continuum: stars)

SN 1986J was one of the most radio luminous supernovae (SNe) ever observed, and one of the few SNe still detectable more than $t=30$ years after the explosion, thus we have been able to follow its evolution for longer than was possible for most other SNe. Its radio brightness and relatively close distance of $D=10 \mathrm{Mpc}$ (Nasa/IPAC Extragalactic Database $\dagger$ ) allowed both resolved very long baseline interferometry (VLBI) images and accurate measurements of its broadband radio spectral energy distribution (SED) to be made.

SN 1986J was unusual in that it was first discovered in the radio, some time after the explosion (Rupen et al. 1987). The best estimate of the explosion epoch is $1983.2 \pm 1.1$ $(t=0$; see Bietenholz et al. 2002). Its optical spectrum showed prominent $\mathrm{H} \alpha$ lines but unusual narrow linewidths, which led Type IIn classification (Rupen et al. 1987). Due to its high radio brightness it was one of the first SNe to be observed with VLBI (e.g. Bartel et al. 1991), and we have continued the observations till the present. We discuss the VLBI observations in a series of papers, Bietenholz et al. $(2002,2010)$ and Bietenholz \& Bartel (2017), which we will refer to as Papers I, II, and III respectively. Paper IV in this series is currently in progress.

We show our latest VLBI image at 5-GHz in Figure 1, observed on 2014 Oct 23, or at $t=31.6$ yr. The observations were made using a global array of VLBI antennas from both the European VLBI Network and the National Radio Astronomy Observatory in the US (see Paper III, for details). A central component dominates the image. Such a central component has not been seen in any other SN (see, e.g. Bietenholz 2014; Bartel

$\dagger$ https://ned.ipac.caltech.edu 


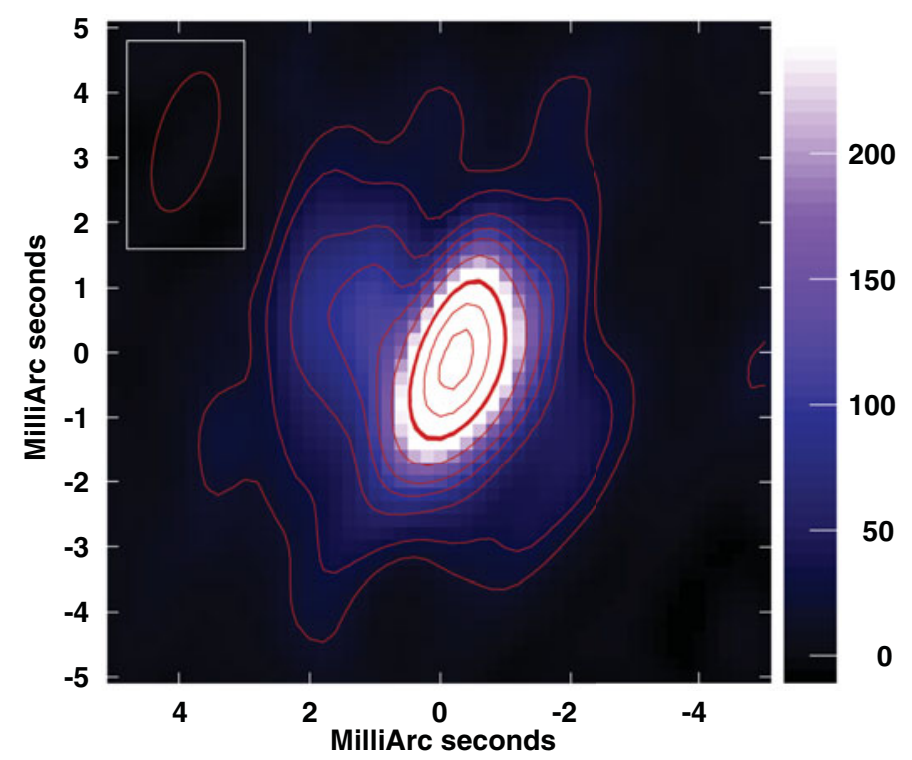

Figure 1. The 5-GHz VLBI image of SN 1986J, made from observations on 2014 Oct. 23, at age $t=31.6 \mathrm{yr}$, reproduced from Paper III. The contours are drawn at $-3,3,5,10,15,20,30, \mathbf{5 0}, 70$ and $90 \%$ of the peak brightness, with the $50 \%$ contour being emphasized. The peak brightness was $617 \mu \mathrm{Jy} \mathrm{beam}^{-1}$, the total CLEANed flux density was $1622 \mu \mathrm{Jy}$, and the background rms brightness was $5.9 \mu \mathrm{Jy}_{\text {beam }}{ }^{-1}$. The colorscale is labelled in $\mu \mathrm{Jy} \mathrm{beam}^{-1}$, and is saturated so as to better show the low-level emission. North is up and east to the left, and the FWHM of the convolving beam is indicated at upper left.
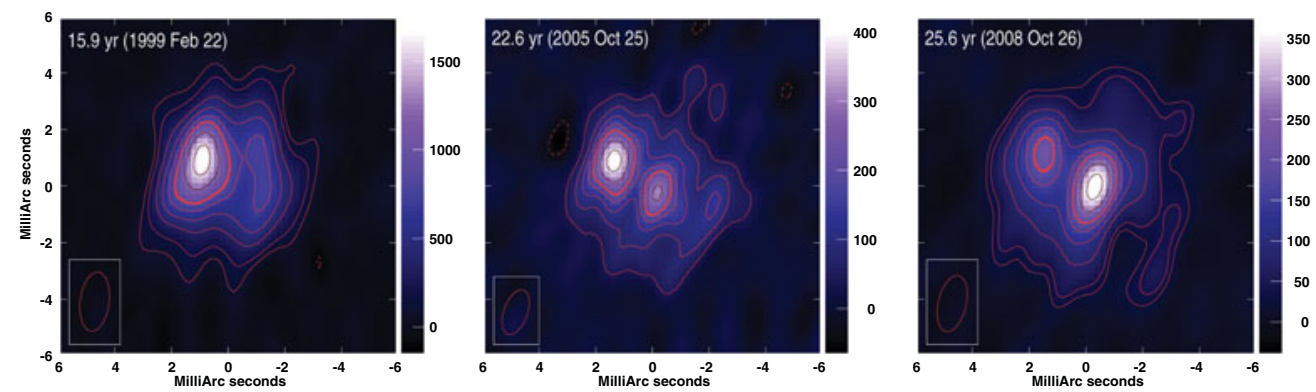

Figure 2. A sequence of 5 -GHz VLBI images of SN 1986J, showing its evolution from $t=15.9 \mathrm{yr}$ to $25.6 \mathrm{yr}$ after the explosion, or 1999 to 2008. Each panel has the lowest contour at $3 \times$ the image background rms, and the remainder at 10,20,30,40, 50,70, and $90 \%$ of the peak brightness, with the $50 \%$ one being emphasized. In each panel, the age and date are given at top left, and the FWHM size of the convolving beam at lower left. North is up and east to the left. The colorscale is in $\mu \mathrm{Jy}_{\text {beam }}{ }^{-1}$. For details of the images, see Paper II. An animation showing the evolution is included in the supplementary materials.

\& Bietenholz 2014), and in particular, no central synchrotron emission has been seen in SN 1987A at cm wavelengths (Zanardo et al. 2014).

The central component is marginally resolved in this image, with a FWHM width of $900_{-500}^{+100} \mu \mathrm{as}$, corresponding to a radius of $r_{\mathrm{comp}}=6.7_{-3.7}^{+0.7} \times 10^{16} \mathrm{~cm}$ (see Paper III, $D=10 \mathrm{Mpc}$ ). Its 5 - $\mathrm{GHz}$ peak brightness in 2014 Oct. was $511 \mu \mathrm{Jy}_{\text {beam }}{ }^{-1}$, corresponding to a spectral luminosity, $L_{\nu}$, of $6 \times 10^{25} \mathrm{erg} \mathrm{s}^{-1} \mathrm{~Hz}^{-1}$, or $\sim 20$ times that of the Crab Nebula. 
In Figure 2, we show a sequence of three $5-\mathrm{GHz}$ images between $t=15.9$ and $25.6 \mathrm{yr}$, showing the emergence of the central component at that frequency. The central component only begins to appear around $t=20 \mathrm{yr}$. In these images, the SN is clearly expanding. The expansion is well described by a powerlaw, with the radius, $r \propto t^{0.69 \pm 0.03}$ (Paper II). The average speed of the outer edge was $5400 \mathrm{~km} \mathrm{~s}^{-1}(t=0$ to $25.6 \mathrm{yr}$, for $D=10 \mathrm{Mpc})$.

A second hot-spot, located in the shell to the north-east, can be seen in the images in Figure 2. At $t=15.9 \mathrm{yr}$, it is the brightest point in the image. It is moving outward, but is also fading in time, and no longer clearly visible by $t=31.6 \mathrm{yr}$.

We measured the proper motions of the shell hotspot and the central component (Paper III), and found that the shell hotspot moves outwards with a mean projected velocity of $2810 \pm 750 \mathrm{~km} \mathrm{~s}^{-1}$ to the NE, which is consistent with it taking part in the homologous expansion of the shell. The central component, on the other hand, is stationary to within the uncertainty of $570 \mathrm{~km} \mathrm{~s}^{-1}$ or $12 \mu \mathrm{as} \mathrm{yr}^{-1}$.

Not only is the appearance of a central component unusual, but so is the evolution of SN 1986J's (SED). The radio SEDs of SNe usually evolve in a fairly predictable way: the flux density, $S_{\nu}$, at frequency, $\nu$, shows an optically-thick inverted spectrum, with positive spectral index $\alpha>1,\left(S_{\nu} \propto \nu^{\alpha}\right)$ up to a turnover frequency, and an optically-thin powerlaw above with $-0.8<\alpha<-0.5$ above the turnover frequency. The absorption at low frequencies is either free-free absorption from intervening thermal material or synchrotron self absorption if the SN is still compact. The turnover frequency evolves downward in time, typically reaching $10 \mathrm{GHz}$ at $t \sim 100 \mathrm{~d}$ (see, e.g. Weiler et al. 2002). There is, however, a large variation in this timescale: for SN 1987A, for example, the turnover frequency reached $10 \mathrm{GHz}$ at $t<1 \mathrm{~d}$ (Turtle et al. 1987) while for SN 2001em (e.g. Bietenholz \& Bartel 2007), as well as for SN 1986J (Weiler et al. 1990), it did not reach $10 \mathrm{GHz}$ till $t \sim 1000 \mathrm{~d}$.

SN 1986J's SED evolved in this fashion, albeit with slow evolution of the turnover frequency, until $t=14.9 \mathrm{yr}$, when an inversion appeared in the spectrum. We show the evolution of the SED in Figure 3. Using multi-frequency phase-referenced VLBI imaging at 5, 8.4 and $15 \mathrm{GHz}$, we showed in Bietenholz et al. (2004) that the spectral inversion was due to the central component, which was not yet promiment at $5-\mathrm{GHz}$ but was already dominant at $15 \mathrm{GHz}$. Since that time, both the inflection point, where the spectrum becomes inverted and the higher frequency turnover point have been moving downward.

The emission from the expanding shell of ejecta in SN 1986J is expected to be optically thin at $\mathrm{GHz}$ frequencies, and to suffer no significant free-free absorption at this late stage (e.g. Weiler et al. 2002). The spectral inversion (at about $2 \mathrm{GHz}$ in 2014) and the higherfrequency turnover (at about $15 \mathrm{GHz}$ in 2014) therefore suggest a source of emission which is still being significantly absorbed by intervening thermal material. In Paper II, we argue that the absorption must be free-free and not synchrotron self-absorption.

We fitted the evolving SED of SN 1986J with a model which had 8 free parameters. The model consisted of two emitting components and some free-free absorption, and the intrinsic flux densities and the amount of absorption all vary with time in a powerlaw fashion. The first component is the emission from the shell, or the expanding shock front, which is unabsorbed and optically thin, and has flux-density

$$
S_{\text {shell }}=S_{0, \text { shell }}\left(\frac{t}{20 \mathrm{yr}}\right)^{b_{\text {shell }}}\left(\frac{\nu}{1 \mathrm{GHz}}\right)^{\alpha_{\text {shell }}}
$$

The second, due to the central component, has an intrinsic SED of

$$
S_{\text {comp }}=S_{0, \text { comp }}\left(\frac{t}{20 \mathrm{yr}}\right)^{b_{\text {com p }}}\left(\frac{\nu}{1 \mathrm{GHz}}\right)^{\alpha_{\mathrm{comp}}}
$$




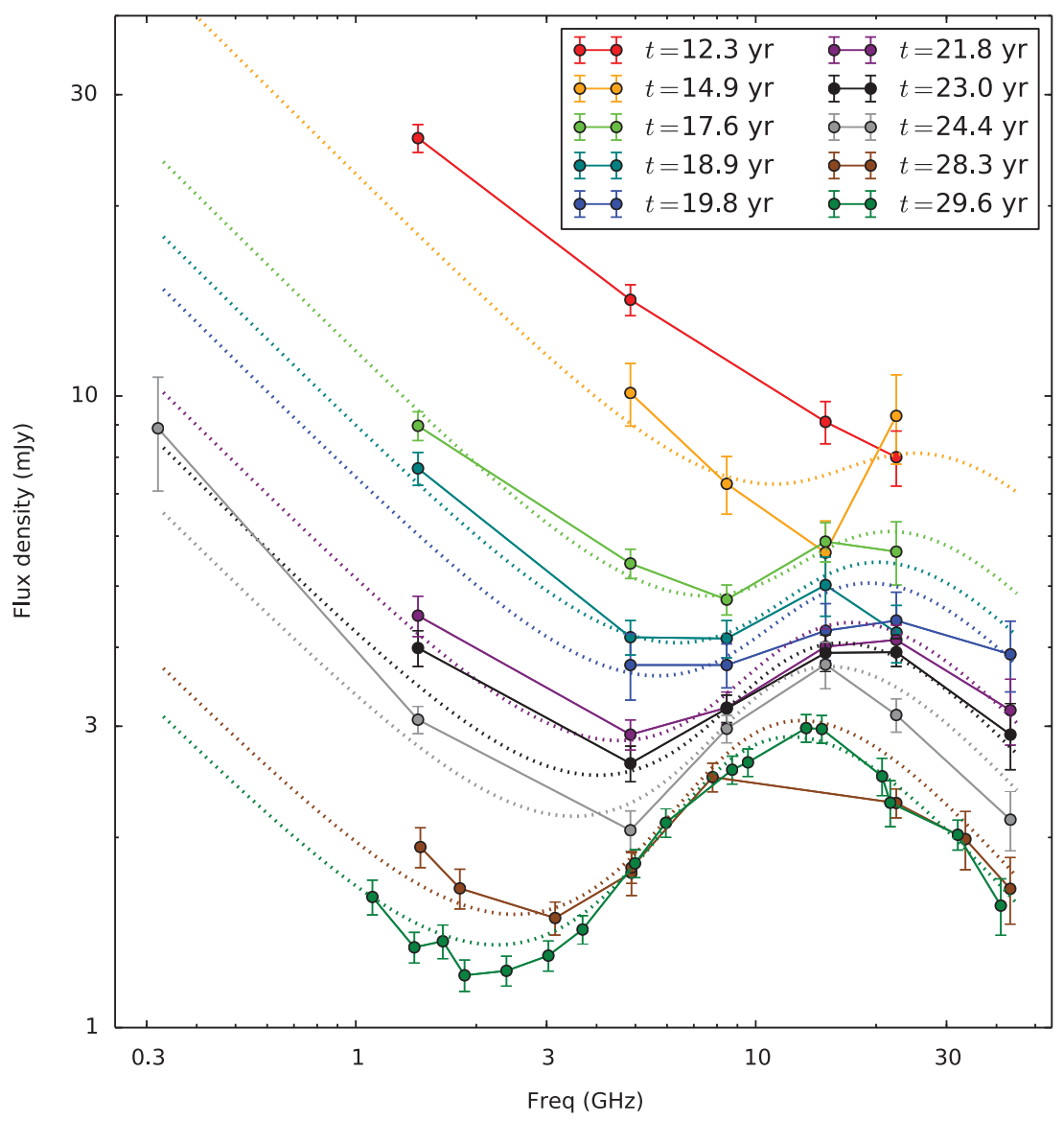

Figure 3. The evolving SED of SN 1986J. The coloured points and associated solid line shows the SED at the indicated age, with the earliest one at the top. The uncertainties are estimated standard errors, including statistical and systematic contributions combined. Data are taken from Paper III and Bietenholz et al. (2004), as well as from the Paper IV (currently in preparation). The dotted lines show a simple model fit to the evolving SED (see text) since the first appearance of the central (inverted spectrum) component at $t=14.9 \mathrm{yr}$. We plot the fitted spectra for each epoch using dotted lines of the same colours as were used for the data points. The model is fairly simple, and fits the SEDs at all epochs simultaneously with only eight free parameters, so the fit to the measurements at any one epoch is only approximate, but the model should give a good overall description of the evolution.

but is free-free absorbed by material with an emission measure, $\mathrm{EM}=\int N_{e}^{2} \cdot d l$, where $N_{e}$ is the electron number density and we assume an electron temperature of $T_{e}=10^{4} \mathrm{~K}$. In our model, the EM is also time-variable and is given by $\mathrm{EM}_{0} \cdot(t / 20 \mathrm{yr})^{b_{\mathrm{EM}}} \mathrm{cm}^{-6} \mathrm{pc}$. In the case of purely external absorption, the inverted part of the spectrum would have a steep frequency dependence, $\propto \nu^{2.1}$, which does not well match our observed SEDs (see Figure 3 and Paper II). Therefore, we take the emitting and the absorbing material to be mixed, in which case the inverted part of the spectrum is $\propto \nu^{\left(\alpha_{\mathrm{comp}}+2.1\right)}$.

We fitted this model to all the VLA flux density measurements after 2014 in Figure 3 (when the central component first appeared) using Bayesian statistics. Details will be given in our upcoming Paper IV. We obtained the following values for the eight free parameters: $S_{0, \text { shell }}=7.07 \pm 0.17 \mathrm{mJy}, b_{\text {shell }}=-3.92 \pm 0.07, \alpha_{\text {shell }}=-0.63 \pm 0.03$, $S_{0, \text { comp }}=61 \pm 17 \mathrm{mJy}, b_{\text {comp }}=-2.07 \pm 0.16, \alpha_{\text {comp }}=-0.76 \pm 0.07, \mathrm{EM}_{0}=(1.64 \pm$ 
$0.20) \times 10^{9} \mathrm{~cm}^{-6} \mathrm{pc}$, and $b_{\mathrm{EM}}=-2.72 \pm 0.26$. We plot the model spectra as dotted lines in Figure 3. The model adequately reproduces the evolving SED, and therefore despite its relative simplicity, we feel gives us some insight into the physics.

The two fitted spectral indices, $\alpha_{\text {shell }}$ and $\alpha_{\text {comp }}$, are very similar, differing by only $1.7 \sigma$ (formally). In view of the relatively simple nature of our model, we do not consider the difference to be significant†. Our measurements are therefore compatible with both the shell and the central component having the same optically-thin spectral index.

The intrinsic flux densities, $S_{\text {comp }}$ and $S_{\text {shell }}$ are both decreasing with time, with $S_{\text {shell }}$ decreasing more rapidly, so that the central component becomes ever more dominant. The amount of absorption affecting the central component is also decreasing with time. At some frequencies, the observed flux density of the central component is increasing with time because the absorption is decreasing more rapidly than the $S_{\text {comp }}$.

In light of these results, what can we say of the nature of the central component? Its high and still increasing brightness, coupled with its stationarity, and long lifetime argue that it really is in or near the three-dimensional centre of SN 1986J, and not associated with the shell and central only in projection.

There are a number of possibilities as to its nature: First, the central component could be due to a young pulsar wind nebula (PWN) formed around a newly born neutron star. Its central location and stationarity are readily explained. The size of the central component is consistent with the expected size of a PWN of $5 \%$ to $10 \%$ of the size of the forward shock. However, its steep intrinsic spectrum and the relatively rapid decay with time of its unabsorbed flux density are not consistent with what is expected of a PWN (see e.g. Gelfand et al. 2009; Chevalier \& Fransson 1992).

Second, since SN 1986J had a massive progenitor, a black hole may have formed. The central component might therefore be due to accretion onto a newly-formed black hole. It is, however, far more radio-luminous than any known stellar-mass black hole system, having an observed $L_{\mathrm{R}} \simeq 3 \times 10^{35} \mathrm{erg} \mathrm{s}^{-1}\left(\nu L_{\nu}\right.$ at $\left.5 \mathrm{GHz}\right)$, with the unabsorbed value being about an order of magnitude larger. Known accreting stellar-mass black hole systems in X-ray binaries, by contrast, have $L_{\mathrm{R}} \lesssim 10^{32} \mathrm{erg} \mathrm{s}^{-1}$ (see, e.g. Körding et al. 2006). The radio luminosity of black hole systems depends on the accretion rate, which might be much higher in the only $\sim 30$ yr old SN 1986J than in the much older X-ray binary systems, and thus produce SN 1986J's unusually high $L_{\mathrm{R}}$. However, in that case, SN 1986J would also be expected to have a high X-ray luminosity, $L_{\mathrm{X}}$. Houck (2005) found that the unabsorbed $L_{\mathrm{X}}$, at $t=20.6 \mathrm{yr}$ was $1.9 \times 10^{39} \mathrm{erg} \mathrm{s}^{-1}(0.5$ to $2.5 \mathrm{keV})$, which is no higher than the brightest X-ray binaries (Körding et al. 2006).

There is a "fundamental plane" of black hole activity, with $L_{\mathrm{R}}, L_{\mathrm{X}}$, and the mass of the black hole all being correlated (see, e.g. Merloni et al. 2003). Even for high masses of $20 M_{\odot}$, this fundamental plane predicts a ratio $L_{\mathrm{R}} / L_{\mathrm{X}}$ lower by several orders of magnitude than is observed for SN 1986J. Furthermore, in Galactic X-ray binaries, high accretion rates seem to quench the radio emission rather than enhance it (e.g. Fender et al. 2010). Therefore, SN 1986J's high radio luminosity, and in particular, its high ratio of $L_{\mathrm{R}} / L_{\mathrm{X}}$, seem discrepant with what is seen in other accreting black hole systems. Given, however, that little is known about newly-born accreting black hole systems inside SN ejecta, a black hole scenario cannot be conclusively ruled out.

Finally, the central component could be explained by SN 1986J's forward shock interacting with a highly anisotropic circumstellar medium (CSM), as was suggested by Chevalier (2012). Such an anisotropic CSM might arise as a result of SN 1986J being

$\dagger$ We also tried a model with only a single intrinsic spectral index characterizing both components, and it provided almost as good a fit with $\alpha=-0.64 \pm 0.03$ 
the second SN in a massive binary system, where there was a period of common envelope interaction between the compact remnant of the first, unobserved, supernova and SN 1986J's progenitor that resulted in very dense and anisotropic mass-loss concentrated in the equatorial plane of the binary. As a result of the dense CSM, the shock takes on something like an hourglass shape, and the central component would represent the narrow waist of the hourglass, the remainder of the shell is due to less equatorial parts of the shock which interact with the more normal supergiant wind of SN 1986J's progenitor. The density of the equatorial CSM could be an order of magnitude higher than that of the remainder (Chevalier 2012). The non-equatorial ejecta initially free-free absorb any emission from the narrow waist, and only after some time do the bulk of the ejecta expand or fragment to the point where the equatorial disk can be seen, at which point the radio central component "turns on." This hypothesis explains the size, central location, low proper motion of the central component, and the near equivalence of $\alpha_{\text {comp }}$ and $\alpha_{\text {shell }}$. Our fitted EM $(1.64 \pm 0.20) \times 10^{9} \mathrm{~cm}^{-6} \mathrm{pc}$ is compatible with what would be expected from a few $M_{\odot}$ of ionized SN ejecta if the ejecta are somewhat centrally concentrated, as is expected to be the case.

At the moment none of the three hypotheses can be definitively ruled out. We are planning more observations to follow the evolution of this fascinating object and hopefully more decisively elucidate the nature of the central component.

\section{References}

Bartel, N. \& Bietenholz, M. F. 2014, in IAU Symposium, Vol. 296, Supernova Environmental Impacts, ed. A. Ray \& R. A. McCray, 53-57

Bartel, N., Rupen, M. P., Shapiro, I. I., Preston, R. A., \& Rius, A. 1991, Nature, 350, 212

Bietenholz, M. 2014, in 12th European VLBI Network Symposium and Users Meeting (2014), published by SISSA, Trieste, ed. A. Tarchi, M. Giroletti, \& L. Feretti, 51

Bietenholz, M. F. \& Bartel, N. 2007, ApJL, 665, L47

-. 2017, ApJ, 839, 10, Paper III

Bietenholz, M. F., Bartel, N., \& Rupen, M. P. 2002, ApJ, 581, 1132, Paper I

-. 2004, Science, 304, 1947

-. 2010, ApJ, 712, 1057, Paper II

Chevalier, R. A. 2012, ApJL, 752, L2

Chevalier, R. A. \& Fransson, C. 1992, ApJ, 395, 540

Fender, R. P., Gallo, E., \& Russell, D. 2010, MNRAS, 406, 1425

Gelfand, J. D., Slane, P. O., \& Zhang, W. 2009, ApJ, 703, 2051

Houck, J. C. 2005, in X-Ray and Radio Connections (eds. L.O. Sjouwerman and K.K Dyer). Published electronically by NRAO, http://www.aoc.nrao.edu/events/xraydio, 4.6.

Körding, E., Falcke, H., \& Corbel, S. 2006, A\&A, 456, 439

Merloni, A., Heinz, S., \& di Matteo, T. 2003, MNRAS, 345, 1057

Rupen, M. P., van Gorkom, J. H., Knapp, et al. 1987, AJ, 94, 61

Turtle, A. J., Campbell-Wilson, D., Bunton, J. D., et al. 1987, Nature, 327, 38

Weiler, K. W., Panagia, N., Montes, M. J., \& Sramek, R. A. 2002, ARAA, 40, 387

Weiler, K. W., Panagia, N., \& Sramek, R. A. 1990, ApJ, 364, 611

Zanardo, G., Staveley-Smith, L., Indebetouw, R., et al. 2014, ApJ, 796, 82 